\title{
Use of anticoagulant or antiplatelet agents is not related to epistaxis in patients undergoing transnasal endoscopy
}

\section{(ㄷ)(1) $\odot$}

\author{
Authors \\ Yoshiya Kobayashi ${ }^{1}$, Yoshinori Komazawa ${ }^{1}$, Mika Yuki ${ }^{1}$, Hitomi Ishitobi ${ }^{1}$, Makoto Nagaoka ${ }^{1}$, Yoshiko Takahashi ${ }^{1}$, \\ Sayaka Nakashima', Toshihiro Shizuku' ${ }^{1}$, Yoshikazu Kinoshita²
}

Institutions

1 Department of Internal Medicine, Izumo City General Medical Center, Shimane, Japan

2 Department of Gastroenterology and Hepatology, Shimane University, Shimane, Japan

submitted 25.7.2017

accepted after revision 15.11.2017

\author{
Bibliography \\ DOI https://doi.org/10.1055/s-0043-124366 | \\ Endoscopy International Open 2018; 06: E104-E110 \\ (c) Georg Thieme Verlag KG Stuttgart · New York \\ ISSN 2364-3722
}

Corresponding author

Yoshiya Kobayashi, Division of Internal Medicine, Izumo City General Medical Center, 613, Nadabun, Izumo, Shimane,

691-0003, Japan

Fax: +81-853-63-4228

yoshiyakbys@gmail.com

\section{ABSTRACT}

Background and study aims Unsedated transnasal endoscopy (UTNE) has become accepted as a safe and tolerable method for upper gastrointestinal tact examinations. Epistaxis is 1 of the major complications of TNE, though its risk factors have not been elucidated. Generally, patients administered an anticoagulant or antiplatelet drug are considered to have an increased risk of epistaxis during TNE. Here, we investigated risk factors of epistaxis in patients undergoing uTNE, with focus on those who received antithrombotic agents.

Patients and methods We enrolled 6860 patients (average age $55.6 \pm 12.97$ years; 3405 males, 3455 females) who underwent UTNE and received the same preparations for the procedure. Epistaxis was evaluated using endoscopic images obtained while withdrawing the scope through the nostril. We also noted current use of medications including anticoagulant or antiplatelet agents prior to the endoscopic examination.

Results Epistaxis occurred in $3.6 \%$ of the enrolled patients (245/6860), and that rate was significantly higher in younger patients (average age $49.31 \pm 11.8$ years for epistaxis group vs. $55.83 \pm 13.0$ years for no epistaxis group, $P<$ $0.01)$ as well as females $(4.78 \%$ vs. $2.35 \%, P<0.01)$. The odds ratio for occurrence of epistaxis was 2.31 (95\% Cl: $1.746-3.167)$ in the younger patients and 2.02 (95\% Cl: $1.542-2.659)$ in females. In contrast, there was no significant difference for rate of epistaxis between patients with and without treatment with an antithrombotic agent (3.0\% vs. $3.6 \%$ ).

Conclusions The rate of epistaxis was higher in younger and female patients. Importantly, that rate was not significantly increased in patients who were administered an antithrombotic agent.

\section{Introduction}

Unsedated transnasal esophagogastroduodenoscopy (UTNE) is a safe alternative transoral approach for endoscopic examination of the upper gastrointestinal tract and shown to be well tolerated by patients [1-3]. Although transoral endoscopy (TOE) has advantages for evaluation of upper gastrointestinal mucosa because of its precise findings shown by chromoendoscopy with magnification and image-enhanced endoscopy (IEE), such as narrow band imaging (NBI), sedation is necessary to reduce TOE-associated discomfort, thus the cost and adverse events associated with use of sedative agents should be consid- ered as limitations of TOE $[4,5]$. On the other hand, use of an ultrathin endoscope is considered to reduce unsedated esophagogastroduodenoscopy (EGD)-associated discomfort.

Currently, TNE is widely accepted and often the preferred choice for daily clinical practice, such as outpatient clinic and annual health checkup examinations, because of its feasibility and tolerability. In addition, uTNE has been reported useful in specific settings, such as for Barrett esophagus surveillance [6], diagnosis of esophageal cancer [7], and examinations of varices in patients with liver cirrhosis [8], and is considered to be associated with a lower level of cardiopulmonary stress [9] and greater patient satisfaction as compared to TOE [10]. In 
Patients who underwent upper Gl examination

$(n=7084)$

Excluded $(n=214)$

- Transoral endoscopy

Transnasal endoscopy $(n=6870)$

$\checkmark$

Enrolled in study

- Including patients with nostril change $(n=32)$

Excluded $(n=10)$

- Unable to insert via transnasal route $(n=10)$

Total number of patients analyzed $(n=6860)$

- Fig. 1 Flowchart of patient selection (from April 2014 to March 2015)

general, the most common complication associated with TNE is epistaxis $[3,11]$, with incidence rates ranging from $1-5 \%$ in several studies, though the symptoms are usually mild and limited $[9,12-16]$. Another study showed that use of a thinner endoscope resulted in a lower rate of epistaxis as compared to scopes with a diameter greater than $5.3 \mathrm{~mm}$ [17]. However, the relationship between epistaxis and patient characteristics in regard to medication administration is unclear. Notably, no study of that association in patients receiving antithrombotic treatment for cerebrovascular or cardiovascular diseases, such as anticoagulant or antiplatelet agents, has been presented, though it is generally considered that administration of anticoagulant or antiplatelet medication is associated with an elevated risk of epistaxis during TNE. In the present study, we investigated risk factors for epistaxis in patients undergoing TNE and receiving antithrombotic treatment.

\section{Patients and methods}

This observational case control study was performed from April 2014 to March 2015 at Izumo City General Medical Center, Shimane, Japan. During that period, we performed upper gastrointestinal examinations in 7084 patients, including those who complained of gastrointestinal symptoms or underwent the examination as part of a medical checkup. Those who underwent upper gastrointestinal endoscopy with a transoral route including route change from nasal to oral were excluded. Finally, a total of 6860 consecutive examined patients were analyzed (average age $55.6 \pm 12.97$ years; 3405 males, 3455 females). Ten were excluded from analysis because of difficulty with insertion through either nostril ( $>$ Fig. 1). Each was given a questionnaire regarding administered medications, including anticoagulant and antiplatelet agents, prior to the endoscopic examination. All procedures were performed by expert gastroenterologists who were well experienced with transnasal insertion of an ultra-thin endoscope. Preparation for TNE was performed in the same manner for all cases, as follows. Initially, the patient was provided a solution containing dimethylpolysiloxane and pronase as premedication to improve mucosal visibility, and also given a naphazoline nitrate spray to both nostrils to prevent nasal edema and hemorrhage. Next, local anesthesia with $2 \%$ lidocaine gel was provided to the nostrils and a pretreatment nasal catheter (14 or $16 \mathrm{Fr}$, Fujifilm, Tokyo, Japan) coated with lidocaine gel was gently inserted into either nostril so that they would not feel pain until the beginning of the endoscopic examination.

Routine examination procedures with an ultrathin endoscope were used, with biopsy samples obtained if clinically indicated. When insertion through the anesthetized nostril was not possible, the insertion route was changed to the other side. If both nostrils showed difficulty with insertion because of nasal pain or narrowness, that patient was excluded from analysis. The diameter of the ultrathin endoscope used was $5.9 \mathrm{~mm}$ (EG-580NW or EG-580NW2, Fujifilm, Tokyo, Japan) or $5.5 \mathrm{~mm}$ (gastrointestinalF-XP $260 \mathrm{~N}$, Olympus, Tokyo, Japan), with the type randomly selected. An inferior nasal meatus or middle nasal meatus insertion route was chosen at the discretion of the attending endoscopist. Following TNE, epistaxis was evaluated during withdrawal of the scope through the nostril and classified as none $(-)$, mild $(+)$, moderate $(++)$, or severe $(+++)$ ( $\triangleright$ Fig.2). If epistaxis did not spontaneously stop, we asked the patient to compress the affected nostril for at least $10-15$ minutes or gave an additional spray of naphazoline. In cases where these procedures were not effective (severe epistaxis), an otolaryngologist was consulted.

Written informed consent was obtained from each patient regarding the transnasal EGD procedure. The study protocol was approved by the ethics committee of Izumo City General Medical Center.

\section{Statistical analysis}

Comparisons between 2 groups were performed using a $x^{2}$ test or Mann-Whitney $U$ test, depending on data distribution. Risk factors related to epistaxis were determined using multivariate logistic regression. A $P$ value $<0.05$ was considered to indicate a statistically significant difference.

\section{Results}

All enrolled patients underwent uTNE with an ultra-thin endoscope and their characteristics are summarized in $>$ Table 1. Epistaxis occurred in $3.6 \%(245 / 6860)$ and the rate was significant higher in younger patients (average age $49.31 \pm 11.8$ years in epistaxis group vs. $55.83 \pm 13.0$ years in no epistaxis group, $P$ $<0.01)$. Furthermore, epistaxis occurred more often in females ( $4.78 \%$ vs. $2.35 \%, P<0.01$ ). There was no significant difference related to the diameter $(5.5$ or $5.9 \mathrm{~mm})$ of the endoscope utilized $(P=0.064)$ ( $\triangleright$ Table 2$)$. Patients receiving antithrombotic agents comprised $3.4 \%(n=233)$ and their average age was significantly older as compared to those not receiving antithrombotic agents $(70.7 \pm 10.5$ vs. $55.1 \pm 12.7$ years, $P<0.01)$. More- 

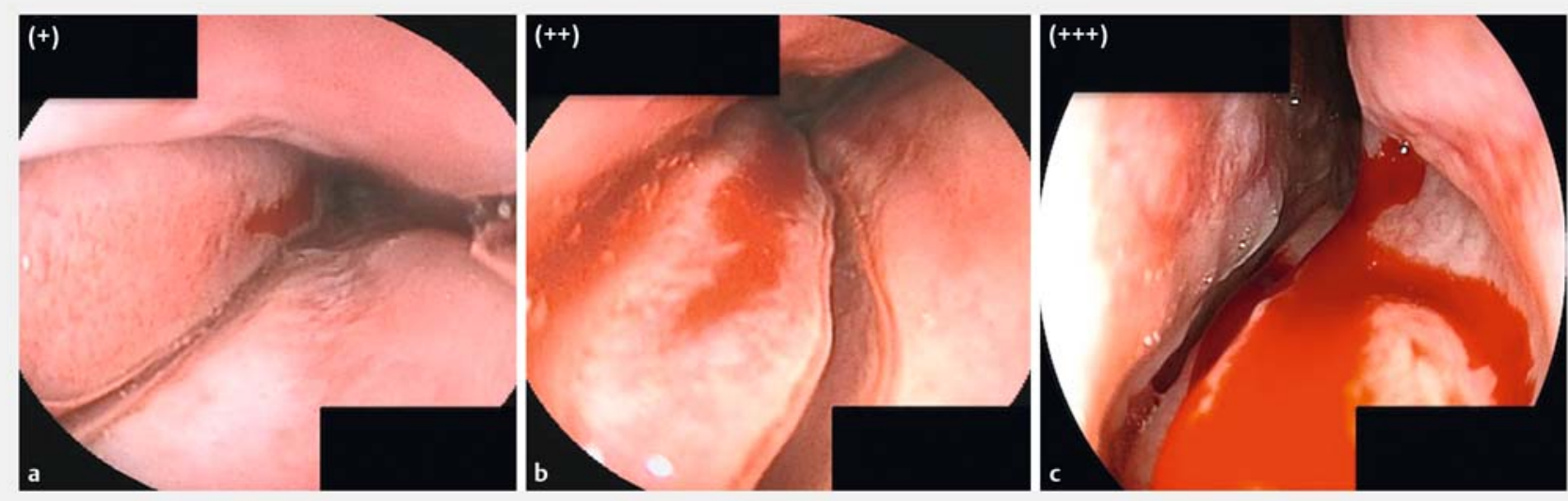

- Fig. 2 Representative images of nasal injuries. A. Mild epistaxis (+). B. Moderate epistaxis (++). C. Severe epistaxis (+++).

- Table 1 Subject characteristics.

\begin{tabular}{|l|l|l|l|}
\hline & Number of patients & Age in years (average \pm SD) & Gender (male/female) \\
\hline Total & 6860 & $55.60 \pm 12.97$ & $3405 / 3455$ \\
\hline & & & n.s. \\
\hline Epistaxis (+) & $245(3.6 \%)$ & $49.31 \pm 11.8$ & $80 / 165^{1}$ \\
\hline Epistaxis (-) & $6615(96.4 \%)$ & $55.83 \pm 13.0$ & $3325 / 3290^{1}$ \\
\hline & & & $147 / 86^{1}$ \\
\hline Antithrombotic treatment & 233 & $70.7 \pm 10.5$ & $3259 / 3368$ \\
\hline No antithrombotic treatment & 6627 & $55.1 \pm 12.7$ & \\
\hline $1 P<0.01$, ns: not significant & & & \\
\hline
\end{tabular}

$>$ Table 2 Rate of epistaxis occurrence.

\begin{tabular}{|l|l|l|l|}
\hline & Number of patients & Age in years (average \pm SD) & Epistaxis rate (\%) \\
\hline Total & 6860 & $55.6 \pm 12.97$ & $245 / 6860(3.6 \%)$ \\
\hline Anti-thrombotic agents & & & $7 / 233(3.00 \%)$ \\
\hline Yes & $\begin{array}{l}\text { P value } \\
(\mathrm{M}: \mathrm{F}=147: 86) \\
(5.5,5.9 \mathrm{~mm}=50: 183)\end{array}$ & $55.1 \pm 12.7$ & 0.635 \\
\hline No & $6627(\mathrm{M}: \mathrm{F}=3258: 3369)$ & $70.7 \pm 10.5$ & $238 / 6627(3.60 \%)$ \\
\hline Gender & & & $80 / 3405(2.35 \%)$ \\
\hline Male & 3405 & $56.0 \pm 12.9$ & $165 / 3455(4.78 \%)$ \\
\hline Female & 3455 & $55.2 \pm 13.1$ & $<0.001$ \\
\hline Endoscope diameter & & $52.31 \pm 10.8$ & 3.50 \\
\hline $5.5 \mathrm{~mm}$ & 1794 & $55.12 \pm 12.3$ & $6.1794(3.84 \%)$ \\
\hline $5.9 \mathrm{~mm}$ & 5066 & & 0.064 \\
\hline
\end{tabular}

over, there was no significant difference for epistaxis rate between those who received and did not receive antithrombotic agents (3.0\% vs. $3.6 \%$ ). All cases of epistaxis were self-limited and $85 \%$ were classified as mild $(+)$, while the remaining were moderate $(++)$ ( $\mathbf{F i g . 2}$ ). None of the enrolled cases showed delayed epistaxis or required consultation with an otolaryngol- 
- Table 3 Multivariate analysis of epistaxis occurring in association with transnasal endoscopy.

\begin{tabular}{|l|l|l|l|}
\hline & Odds ratio & $\mathbf{9 5 \% C l}$ & \multicolumn{1}{c|}{ value } \\
\hline Gender (female) & 2.0249 & $1.542-2.659$ & $<0.0001$ \\
\hline Age (younger) & 2.3139 & $1.746-3.167$ & $<0.0001$ \\
\hline Anticoagulation therapy & 1.4180 & & 0.3821 \\
\hline Endoscope & 1.0151 & & 0.7639 \\
\hline
\end{tabular}

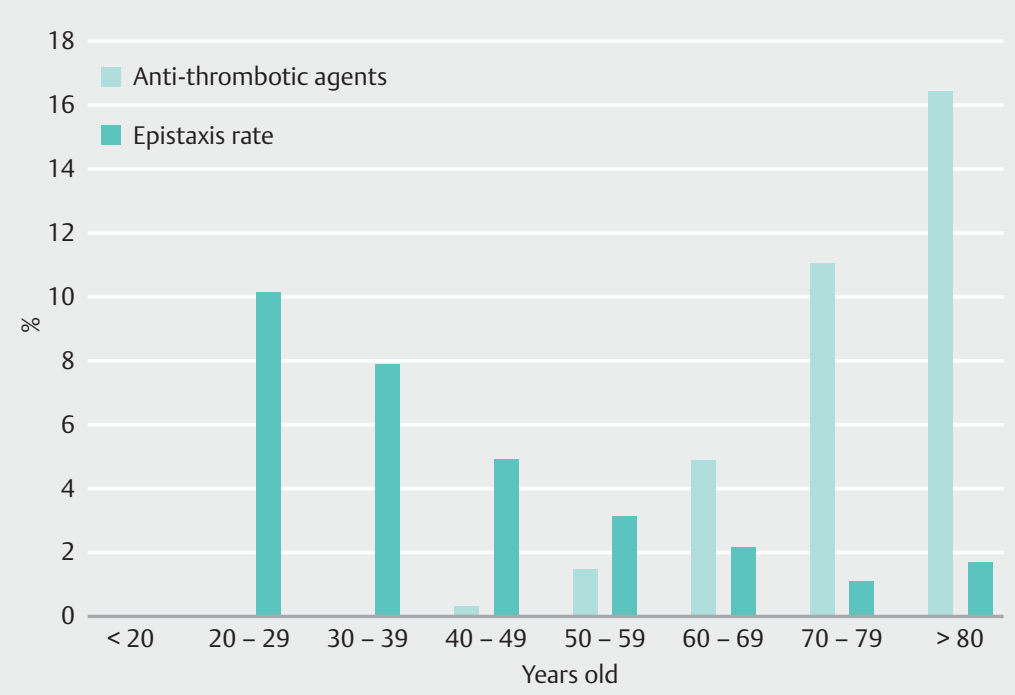

Fig.3 Patients receiving antithrombotic agents were older, while epistaxis occurred more frequently in younger patients.

ogist. Multivariate logistic regression revealed that the risk factors for epistaxis were age and gender, with an odds ratio of 2.31 (95\% Cl: $1.746-3.167)$ for younger age and $2.02(95 \% \mathrm{Cl}$ : $1.542-2.659$ ) for females ( $>$ Table 3 ).

\section{Discussion}

Improvements in ultra-thin endoscopes have allowed patients to undergo safe and tolerable EGD procedures. Several investigations have shown that TNE is more preferable as compared to $\operatorname{TOE}[9,10]$, though fewer reports regarding complications with TNE have been presented, especially of epistaxis occurring in patients receiving antithrombotic treatment. In the present study, epistaxis occurred in $3.6 \%$ of the 6860 enrolled patients and, most importantly, that rate was approximately equal between those who were and were not receiving antithrombotic agents. Previous studies have reported epistaxis rates ranging from $1-5 \%$, the vast majority of which were self-limited [ 9 , 12 -16], the same as seen in the present study, while none of our patients required otolaryngology treatment following the procedure. Nevertheless, little is known regarding risk factors for epistaxis associated with TNE, especially in patients who are receiving antithrombotic agents. Mori et al. [18] investigated nasal bleeding sites, though the characteristics of their patients were not presented. As noted above, no investigation in regard to the relationship between epistaxis and patient baseline characteristics including administered medications has been reported. To the best of our knowledge, this is the first report of the involvement of epistaxis in individuals receiving antithrombotic agents. Among the present cohort, patients who were prescribed antithrombotic agents were older than those who did not take those agents ( $\downarrow$ Table 1 ), while epistaxis occurred frequently in younger patients ( $>$ Fig.3). Accordingly, our results clearly demonstrated that TNE is a preferable method for an upper gastrointestinal examination in elderly patients with cerebrovascular or cardiovascular diseases who are receiving antithrombotic agents. In addition, they suggest that withdrawal of antithrombotic agents prior to TNE is unnecessary and not a concern in regard to epistaxis occurrence.

Antithrombotic medications used by the present patients were antiplatelet agents in 72 (aspirin, clopidogrel, cilostazol, others), an anticoagulant in 21 (warfarin), direct oral anticoagulant agents (DOACs) in 14, and others in 12, while those details were unknown in 114 . There was no significant difference in regard to epistaxis rate among the prescribed drugs, though our data are insufficient because details were unclear in up to half of the cases. In our previous study conducted in 2010, we evaluated the effects of occurrence of epistaxis during a TNE procedure in association with endoscope diameter, gender, age, and type of anticoagulant or antiplatelet medication using 
- Table 4 Odds ratio of epistaxis in patients who receiving anti-thrombotic agents. (total 4251 patients, 195 receiving anti-thrombotic agents)

\begin{tabular}{|l|l|l|l|l|l|}
\hline Antithrombotic agents & Number of patients & Epistaxis rate (\%) & Odds ratio & 95\%Cl & \\
\hline Total & 195 & 7.2 & & $0.564-4.454$ \\
\hline Warfarin & 47 & 8.5 & 1.585 & $0.48-2.556$ \\
\hline Aspirin & 98 & 6.1 & 1.107 & 0.468 \\
\hline Others & 50 & 6.0 & 1.094 & $0.395-3.034$ \\
\hline
\end{tabular}

multivariate analysis in 4251 patients (average age $57.7 \pm 15.1$ years; males 2329, females 1922). Those results were similar to the present in regard to each type of medication. In the present study, patients who were receiving antithrombotic treatment (warfarin 47 , aspirin 98 , others 50 ) comprised $4.6 \%$ of the total, with no differences in regard to type of anticoagulant or antiplatelet medication related to occurrence of epistaxis $(\triangleright$ Table 4). Interestingly, the rate of epistaxis in patients with anti-thrombotic treatment in the present study performed in 2015 was reduced as compared to that in our study performed in 2010. We presume that this result was due, at least in part, to premedication with a naphazoline nitrate spray given for a longer prior to inserting the nasal catheter, which may have helped to prevent nasal edema and hemorrhage in the nostrils.

In previously presented guidelines $[19,20]$, diagnostic EGD, mucosal biopsy, and biliary pancreatic stenting are included as procedures with a low risk for gastrointestinal bleeding. It is well known clinically that inattentive interruption of antithrombotic administration can cause a fatal thromboembolic event. In light of the present findings, we consider that TNE is the preferred method for an upper gastrointestinal screening examination, including a procedure to obtain biopsy samples, even in patients for whom it is too dangerous to discontinue antithrombotic agents due to the high risk of a thromboembolic event. As recommended in those guidelines, the probability of a thromboembolic event related to temporary interruption of an antithrombotic agent for an endoscopic procedure is dependent on indication for antithrombotic therapy and individual patient characteristics. For example, clinical estimation of the risk of a cerebrovascular accident occurring in patients with non-valvular atrial fibrillation is generally evaluated using the CHA2DS2-VASc index [21]. With the present aging of society, ischemic heart disease and stroke are major causes of death, thus the number of gastrointestinal screening examinations and therapeutic endoscopy procedures for patients who are being treated with antithrombotic medication will likely increase. To prevent fatal thromboembolic events, careful management must be provided in association with an endoscopic procedure.

For the 6860 patients who underwent TNE in the current study, the rate of epistaxis was similar to that in previous investigations and all of our cases were self-limited. We consider that these results are reliable because of the large number of cases involved. In previous studies of patients receiving aspirin, clopidogrel, or warfarin who underwent a biopsy, there was no increased risk of hemorrhage $[22,23]$. Several guidelines indicate the risk of gastrointestinal bleeding $[19,20]$, whereas epistaxis associated with TNE has not been studied prior to the present investigation. Thus, transoral endoscopy with a sedative agent is more popular in Western countries and TNE is not recognized as an alternative to TOE for routine endoscopic examinations. Furthermore, use of an ultrathin endoscope is not appropriate for a therapeutic procedure because of the narrow working channel. On the other hand, in Asia, particularly Japan, TNE has become common because of its convenience for endoscopists and acceptability by patients. In addition, TNE can be the first choice for an upper gastrointestinal screening examination under an unsedated condition, since visualization is comparable to that with a standard endoscope due to recent developments in endoscopic technology.

Our multivariate analysis findings showed that younger age (<65 years) and female gender were significant risk factors for epistaxis in patients who underwent TNE. Previously, female gender and age were reported to be independent significant predictors of increased visual analog scale (VAS) scores for transnasal insertion [24,25]. Generally, TNE is preferred for younger patients, with transoral insertion better for aged males, as younger patients are likely have a greater capacity to avoid gag reflex. Also, there might be gender differences in regard to diminished gagging reflex and nasal pain associated with aging. Thus, we propose that nasal pain and epistaxis are related, because of the generally narrower nasal cavities in younger and female individuals. Some reports have noted that pain during transnasal insertion did not alter the higher level of patient satisfaction with TNE [26-28], while a recent study showed that the diagnostic effectiveness of TNE is comparable to that of standard endoscopy [9]. Hence, TNE may be a good choice for upper gastrointestinal screening with high tolerability, though complications of epistaxis and nasal pain must be considered.

There are several limitations to this study. First, it was performed at a single institution. However, a large number of cases was analyzed and there may be no significant technical differences between institutions, because the TNE procedures including preparation are commonly conducted by nurses and gastroenterologists throughout Japan. Second, patient adherence to their prescribed antithrombotic medication was not closely confirmed, and laboratory data such as prothrombin time and international normalized ratio (INR) were not routinely determined in those taking warfarin prior to TNE. At present, details regarding occurrence of epistaxis in individuals receiving DOACs are largely unknown and only a few of the present 
patients in this study were were receiving those. Third, we did not obtain data for epistaxis rate related to insertion route, such as inferior nasal meatus (INM) and middle nasal meatus (MNM). A previous study presented findings of anterior meatoscopy procedures and reported that the endoscopic meatus scoring scale was more reliable than a sniff test for predicting nasal patency [29]. However, we consider that the utility of this scoring method for reducing epistaxis and nasal pain is limited, which is why we do not clearly distinguish regarding common nasal meatus (CNM) and nearly all endoscopists in Japan regarded that to be similar to MNM. Thus, we think that there is little advantage for distinguishing CNM from MNM. Finally, there were no data available for patients who had bleeding tendency, such as liver cirrhosis and hematological disease cases, thus we were unable to analyze the effectiveness of TNE for such cases.

In summary, our findings demonstrated a relationship between epistaxis and individual patient factors, especially administration of antithrombotic medications. We consider that TNE can be performed safely for an upper gastrointestinal examination in elderly patients without discontinuation of antithrombotic administration.

\section{Conclusion}

In subjects who underwent a TNE procedure, epistaxis occurred in $3.6 \%$, though that rate was higher in younger patients and females. Importantly, the rate of epistaxis was not significantly increased in those receiving antiplatelet or anticoagulant agents. We concluded that TNE can be performed safely even in elderly patients without cessation of antithrombotic agents.

\section{Acknowledgements}

We thank Dr. Kinoshita, Professor at the Department of Gastroenterology and Hepatology, Shimane University, for reviewing the manuscript.

\section{Competing interests}

None

\section{References}

[1] Yagi ], Adachi K, Arima $\mathrm{N}$ et al. A prospective randomized comparative study on the safety and tolerability of transnasal duodenoscopy. Endoscopy 2005; 37: 1226 - 1231

[2] Yuki M, Amano Y, Komazawa Y et al. Unsedated transnasal small-caliber esophagogastroduodenoscopy in elderly and bedridden patients. World J Gastroenterol 2009; 15: 5586- 5591

[3] Tatsumi Y, Harada A, Matsumoto T et al. Current status and evaluation of transnasal esophagogastroduodenoscopy. Digestive Endosc 2009; 21: $141-146$

[4] Waring JP, Baron TH, Hirota WK et al. Guidelines for conscious sedation and monitoring during gastrointestinal endoscopy. Gastrointest Endosc 2003; 58: $317-322$
[5] Trevisani L, Sartori S, Gaudenzi P et al. Upper gastrointestinal endoscopy: are preparatory interventions or conscious sedation effective? A randomized trial World J Gastroenterol 2004; 10: 3313-3317

[6] Shariff MK, Bird-Lieberman EL, O'Donovan M et al. Randomized crossover study comparing efficacy of transnasal endoscopy with that of standard endoscopy to detect Barrett's esophagus. Gastrointest Endosc 2012; 75: 954-61

[7] Huang YC, Lee YC, Tseng PH et al. Regular screening of esophageal cancer for 248 newly diagnosed hypopharyngeal squamous cell carcinoma by unsedated transnasal esophagogastroduodenoscopy. Oral Oncol 2016; 55: 55-60

[8] Choe WH, Kim JH, Ko SY et al. Comparison of transnasal small-caliber vs. peroral conventional esophagogastroduodenoscopy for evaluating varices in unsedated cirrhotic patients. Endoscopy 2011; 43: 649-656

[9] Ai ZL, Lan CH, Fan LL et al. Unsedated transnasal upper gastrointestinal endoscopy has favorable diagnostic effectiveness, cardiopulmonary safety, and patient satisfaction compared with conventional or sedated endoscopy. Surg Endosc 2012; 26: 3565-3572

[10] Kadayifci A, Atar M, Parlar S et al. Transnasal endoscopy is preferred by transoral endoscopy experienced patients. J Gastrointestin Liver Dis 2014; $23: 27-31$

[11] Maffei M, Dumonceau JM. Transnasal esogastroduodenoscopy (EGD): comparison with conventional EGD and new applications. Swiss Med Wkly 2008; 138: $658-664$

[12] Cho S, Arya N, Swan K et al. Unsedated transnasal endoscopy: a Canadian experience in daily practice. Can J Gastroenterol 2008; 22: $243-246$

[13] Stroppa I, Grasso E, Paoluzi OA et al. Unsedated transnasal versus transoral sedated upper gastrointestinal endoscopy: a one-series prospective study on safety and patient acceptability. Dig Liver Dis 2008; 40: $767-775$

[14] Lee SY, Kawai T. Transnasal route: new approach to endoscopy. Gut Liver 2008; 2: 155-165

[15] Watanabe H, Watanabe N, Ogura R et al. A randomized prospective trial comparing unsedated endoscopy via transnasal and transoral routes using 5.5-mm video endoscopy. Dig Dis Sci 2009; 54: 2155 2160

[16] Trevisani L, Cifalà V, Sartori S et al. Unsedated ultrathin upper endoscopy is better than conventional endoscopy in routine outpatient gastroenterology practice: a randomized trial. World J Gastroenterol 2007; 13: 906-911

[17] Maffei M, Dumonceau JM. Transnasal esophagogastroduodenoscopy (EGD): comparison with conventional EGD and new applications. Swiss Med Wkly 2008; 138: 658-664

[18] Mori A. Reporting the how, where, and grading of nasomucosal injury after transnasal endoscopy (Letter reply to Hu). Endoscopy 2010; 42: 689

[19] Acosta RD, Abraham NS, Chandrasekhara V et al. The management of antithrombotic agents for patients undergoing gastrointestinal endoscopy. Gastrointest Endosc 2016; 83: 3-16

[20] Veitch AM, Vanbiervliet G, Gershlick AH et al. Endoscopy in patients on antiplatelet or anticoagulant therapy, including direct oral anticoagulants: British Society of Gastroenterology (BSG) and European Society of Gastrointestinal Endoscopy (ESGE) guidelines. Gut 2016; 65: $374-389$

[21] Lip GY, Nieuwlaat R, Pisters R et al. Refining clinical risk stratification for predicting stroke and thromboembolism in atrial fibrillation using a novel risk factor-based approach: the euro heart survey on atrial fibrillation. Chest 2010; 137: 263-272

[22] Ono S, Fujishiro M, Kodashima $S$ et al. Evaluation of safety of endoscopic biopsy without cessation of antithrombotic agents in Japan. J Gastroenterol 2012; 47: $770-774$ 
[23] Whitson M], Dikman AE, von Althann C et al. Is gastroduodenal biopsy safe in patients receiving aspirin and clopidogrel: a prospective, randomized study involving 630 biopsies. J Clin Gastroenterol 2011; 45: $228-233$

[24] Ono S, Niimi K, Fujishiro M et al. Evaluation of preferable insertion routes for esophagogastroduodenoscopy using ultrathin endoscopes. World J Gastroenterol 2014; 20: 5045 - 5050

[25] Ono S, Niimi K, Fujishiro M et al. Ultrathin endoscope flexibility can predict discomfort associated with unsedated transnasal esophagogastroduodenoscopy. World J Gastrointest Endosc 2013; 5: 346-351

[26] Preiss C, Charton JP, Schumacher B et al. A randomized trial of unsedated transnasal small-caliber esophagogastroduodenoscopy (EGD) versus peroral small-caliber EGD versus conventional EGD. Endoscopy 2003; 35: 641-646

[27] Cheung J, Bailey R, Veldhuyzen van Zanten S et al. Early experience with unsedated ultrathin $4.9 \mathrm{~mm}$ transnasal gastroscopy: a pilot study. Can J Gastroenterol 2008; 22: $917-922$

[28] Murata A, Akahoshi K, Sumida Y et al. Prospective randomized trial of transnasal versus peroral endoscopy using an ultrathin videoendoscope in unsedated patients. J Gastroenterol Hepatol 2007; 22: $482-$ 485

[29] Hu CT. Anterior meatuscopy is more reliable than a sniff test for predicting nasal patency before transnasal endoscopy. Endosc Int Open 2015; 3: E538-E546 